\title{
FACES WITH GIVEN DIRECTIONS IN ANISOTROPIC POISSON HYPERPLANE MOSAICS
}

\author{
DANIEL HUG, ${ }^{*}$ Karlsruhe Institute of Technology \\ ROLF SCHNEIDER, ${ }^{* *}$ Albert-Ludwigs-Universität Freiburg
}

\begin{abstract}
For stationary Poisson hyperplane tessellations in $d$-dimensional Euclidean space and a dimension $k \in\{1, \ldots, d\}$, we investigate the typical $k$-face and the weighted typical $k$-face (weighted by $k$-dimensional volume), without isotropy assumptions on the tessellation. The case $k=d$ concerns the previously studied typical cell and zero cell, respectively. For $k<d$, we first find the conditional distribution of the typical $k$-face or weighted typical $k$-face, given its direction. Then we investigate how the shapes of the faces are influenced by assumptions of different types: either via containment of convex bodies of given volume (including a new result for $k=d$ ), or, for weighted typical $k$-faces, in the spirit of D. G. Kendall's asymptotic problem, suitably generalized. In all these results on typical or weighted typical $k$-faces with given direction space $L$, the Blaschke body of the section process of the underlying hyperplane process with $L$ plays a crucial role.
\end{abstract}

Keywords: Poisson hyperplane tessellation; volume-weighted typical face; typical face; conditional distribution; Blaschke body; shape

2010 Mathematics Subject Classification: Primary 60D05

\section{Introduction}

A stationary Poisson hyperplane mosaic in Euclidean space $\mathbb{R}^{d}$ gives rise to interesting random polytopes. The zero cell is the almost surely unique cell containing a given point, without loss of generality, the origin of $\mathbb{R}^{d}$. Typical cells are obtained, heuristically, by picking out cells at random, possibly with weights; a precise definition can be given with the aid of Palm distributions (see Section 2). The investigation of asymptotic shapes of such random polytopes was initiated by a well-known problem of David Kendall. He asked (see the formulation in the foreword to the first edition of [14]) whether the zero cell of a stationary, isotropic Poisson line process in the plane, under the condition of large area, must be approximately circular, with high probability. After an affirmative answer had been given by Kovalenko [7], extensions of the problem to higher dimensions and without the assumption of isotropy were treated. In the anisotropic case, it turned out that the so-called Blaschke body, an auxiliary centrally symmetric convex body constructed from the directional distribution of the underlying hyperplane process, governs the asymptotic shape. In [5], a suitable measure of shape deviation from the Blaschke body was defined, and the following was shown. The probability that the shape of the zero

Received 30 April 2010; revision received 24 January 2011.

* Postal address: Department of Mathematics, Karlsruhe Institute of Technology, D-76128 Karlsruhe, Germany.

Email address: daniel.hug@kit.edu

** Postal address: Mathematisches Institut, Albert-Ludwigs-Universität Freiburg, Eckerstr. 1, D-79104 Freiburg, Germany. Email address: rolf.schneider@math.uni-freiburg.de 
cell or the shape of the typical cell of a stationary Poisson hyperplane mosaic in $\mathbb{R}^{d}$, under the condition that the volume of the cell is at least $a>0$, deviates from the shape of the Blaschke body by more than some prescribed bound is exponentially small with increasing $a$. Various modifications of Kendall's problem are treated in [2], [3], and [6].

There are also other manifestations of the phenomenon that, for the shape of the cells of stationary Poisson hyperplane tessellations, under suitable assumptions, the Blaschke body of its underlying Poisson hyperplane process plays a crucial rôle. Let $K \subset \mathbb{R}^{d}$ be a convex body containing the origin and of given positive volume. Then the probability that the zero cell contains $K$ is maximal if and only if $K$ is homothetic to the Blaschke body (see [13, Theorem 10.4.11]). Below we prove a (less immediate) analogue of this result for the typical cell (Theorem 2 of Section 4).

The notions of typical cell and zero cell can be generalized to faces of lower dimensions in the mosaic, in the form of typical $k$-faces and $k$-volume weighted typical $k$-faces. The extension of the mentioned results to typical or weighted typical $k$-faces, for $k<d$, meets with the difficulty that properties of lower-dimensional faces in the mosaic depend on their directions, except if the mosaic is isotropic. By the direction of a $k$-dimensional convex body we understand here the linear subspace that is a translate of the affine hull of the body. A version of Kendall's problem for typical $k$-faces was treated in [4], where the condition of large $k$-volume was supplemented by the condition that the direction of the typical $k$-face is in a small neighbourhood of a prescribed direction. In the following, we deal with typical $k$-faces of a given direction. Since, in general, these occur only with probability 0 , we consider the regular conditional probability distribution of the typical $k$-face (or weighted typical $k$-face), given that its direction is a prescribed linear subspace $L$. Our first main result (Theorem 1 of Section 3 ) says that this conditional distribution is equal to the distribution of the typical cell (respectively, the zero cell) of a stationary Poisson hyperplane process in $L$, whose intensity and directional distribution are determined in a simple way from those of the original hyperplane process. We see this fact as a further remarkable consequence of the independence properties of Poisson processes. It allows us to extend the mentioned results for zero and typical cells to (weighted) typical $k$-faces with given direction (Theorem 3 of Section 4, and Theorems 4 and 5 of Section 5).

\section{Preliminaries}

First, we fix some notation and collect the fundamental facts about stationary Poisson hyperplane tessellations (or mosaics-both terms are used synonymously). General introductions to random tessellations can be found in [10], Chapter 10 of [13], and [14].

We work in $d$-dimensional Euclidean vector space $\mathbb{R}^{d}(d \geq 2)$, with origin $\boldsymbol{o}$, scalar product $\langle\cdot, \cdot\rangle$, and norm $\|\cdot\|$. Its unit sphere is denoted by $\mathbb{S}^{d-1}$, the Grassmannian of $k$-dimensional linear subspaces of $\mathbb{R}^{d}$ by $G(d, k)$, and the affine Grassmannian of $k$-dimensional affine subspaces of $\mathbb{R}^{d}$ by $A(d, k)$. These spaces carry their standard topologies. For $L \in G(d, k)$, the set $\mathbb{S}_{L}:=\mathbb{S}^{d-1} \cap L$ is the unit sphere in $L$. By $\mathcal{K}$ we denote the space of convex bodies (nonempty, compact, convex sets) in $\mathbb{R}^{d}$, endowed with the Hausdorff metric. (Notions and results from convex geometry that are not explained here can be found in [11].) For a convex body $K$, the number $V_{k}(K)$ is its $k$ th intrinsic volume, and we note that, for $\operatorname{dim} K=k$, this is the $k$-dimensional volume of $K$. By $\mathscr{B}(T)$ we denote the Borel $\sigma$-algebra of a topological space $T$. As usual, we identify a simple counting measure on a topological space with its support. Accordingly, the realizations of a point process which is simple and locally finite (as is always assumed in the following) are also treated as locally finite sets. 
Throughout this paper, we assume that $X$ is a stationary Poisson hyperplane process in $\mathbb{R}^{d}$, that is, a Poisson point process in the space $A(d, d-1)$ of hyperplanes, with a distribution that is invariant under translations. The underlying probability space is denoted by $(\Omega, A, P)$, and the mathematical expectation by $\mathrm{E}$. The intensity measure $\Theta=\mathrm{E} X(\cdot)$ of $X$ has a representation (see, e.g. [13, Equation (4.33)])

$$
\Theta(A)=2 \gamma \int_{\mathbb{S}^{d-1}} \int_{0}^{\infty} \mathbf{1}_{A}(H(\boldsymbol{u}, t)) \mathrm{d} t \varphi(\mathrm{d} \boldsymbol{u})
$$

for $A \in \mathcal{B}(A(d, d-1))$, where we parametrize hyperplanes which do not contain $\boldsymbol{o}$ by

$$
H(\boldsymbol{u}, t)=\left\{\boldsymbol{x} \in \mathbb{R}^{d}:\langle\boldsymbol{x}, \boldsymbol{u}\rangle=t\right\}
$$

with $\boldsymbol{u} \in \mathbb{S}^{d-1}$ and $t>0$. We assume that $\gamma$, the intensity of $X$, is positive and that $\varphi$, the directional distribution of $X$, is an even probability measure on the sphere $\mathbb{S}^{d-1}$ which is not concentrated on any great subsphere (i.e. on a set $\mathbb{S}^{d-1} \cap L$, where $L$ is a $(d-1)$-dimensional linear subspace of $\mathbb{R}^{d}$ ).

Owing to the latter assumption, the hyperplane process $X$ induces a random tessellation or mosaic, in the obvious way. We denote this random mosaic (i.e. as in [13, Chapter 10], the particle process of its cells) by $X^{(d)}$, and the process of its $k$-dimensional faces by $X^{(k)}$ for $k=0, \ldots, d-1$. For the definition of grain distributions, we need a centre function (see [13, p. 110]), and we use here the Steiner point (see [13, Equation (14.28)]). Being a stationary particle process, $X^{(k)}$ has a grain distribution with respect to the Steiner point, and a random polytope with this distribution is called the typical $k$-face of the random mosaic $X^{(d)}$ (as in [13]). Equivalently, we can define the random measure $N_{k}$ assigning mass 1 to each Steiner point of a $k$-face of the mosaic and then use the Palm distribution $\mathrm{P}_{N_{k}}^{0}$ of $X$ with respect to $N_{k}$. If $Y$ is a hyperplane process with distribution $\mathrm{P}_{N_{k}}^{0}$ then its induced mosaic has almost surely a unique $k$-face containing $\boldsymbol{o}$. This defines a random polytope which is stochastically equivalent to the typical $k$-face of $X^{(d)}$. The same procedure, with $N_{k}$ replaced by the random measure defined by the $k$-dimensional Hausdorff measure restricted to the $k$-skeleton of $X^{(d)}$ (i.e. the union of its $k$-faces), leads to the $k$-volume weighted typical $k$-face of $X^{(d)}$. Instead of ' $k$-volume weighted typical $k$-face' we briefly say 'weighted typical $k$-face'. We denote the typical $k$-face by $Z^{(k)}$ and the weighted typical $k$-face by $Z_{0}^{(k)}$. The distributions of both random polytopes are connected by the relation

$$
\mathrm{E} f\left(Z_{0}^{(k)}\right)=\frac{1}{\mathrm{E} V_{k}\left(Z^{(k)}\right)} \mathrm{E}\left[f\left(Z^{(k)}\right) V_{k}\left(Z^{(k)}\right)\right],
$$

which holds for every translation invariant, nonnegative, measurable function $f$ on the space of $k$-dimensional polytopes. (More details can be found in, e.g. [1] and [12]). The typical $d$-face, $Z^{(d)}$, is briefly denoted by $Z$ and called the typical cell. The weighted typical $d$-face is stochastically equivalent to the zero cell of $X^{(d)}$, which is defined by

$$
Z_{0}:=\bigcap_{H \in X} H^{-} .
$$

Here $H^{-}$denotes the (for $\boldsymbol{O} \notin H$ unique) closed half-space bounded by $H$ that contains $\boldsymbol{o}$.

The investigation of these random polytopes requires a few more constructions from the theory of hyperplane processes. For $k \in\{0, \ldots, d-2\}$, the intersection process of $X$ of order $d-k$ is obtained by intersecting any $d-k$ hyperplanes of $X$ which have linearly independent 
normal vectors. It is a stationary process of $k$-flats and is denoted by $X_{d-k}$. According to [13, Theorem 4.4.8], its intensity $\gamma_{d-k}$ and its directional distribution $\mathrm{Q}_{d-k}$ are given by

$$
\begin{gathered}
\gamma_{d-k} \mathrm{Q}_{d-k}(A)=\frac{\gamma^{d-k}}{(d-k) !} \int_{\left(\mathbb{S}^{d-1}\right)^{d-k}} \mathbf{1}_{A}\left(\boldsymbol{u}_{1}^{\perp} \cap \ldots \cap \boldsymbol{u}_{d-k}^{\perp}\right) \nabla_{d-k}\left(\boldsymbol{u}_{1}, \ldots, \boldsymbol{u}_{d-k}\right) \\
\times \varphi^{d-k}\left(\mathrm{~d}\left(\boldsymbol{u}_{1}, \ldots, \boldsymbol{u}_{d-k}\right)\right)
\end{gathered}
$$

for $A \in \mathcal{B}(G(d, k))$. Here $\boldsymbol{u}^{\perp}$ denotes the orthogonal complement of the linear subspace spanned by $\boldsymbol{u}$, and $\nabla_{m}\left(\boldsymbol{u}_{1}, \ldots, \boldsymbol{u}_{m}\right)$ is the $m$-dimensional volume of the parallelepiped spanned by $\boldsymbol{u}_{1}, \ldots, \boldsymbol{u}_{m}$. In addition, $\mathrm{Q}_{1}:=\varphi$.

Let $k \in\{1, \ldots, d-1\}$ and $L \in G(d, k)$. The section process of $X$ with $L$ is obtained by intersecting $L$ with each hyperplane of $X$ which is in general position with respect to $L$, that is, satisfies $\operatorname{dim}(L \cap H)=k-1$. This results in a stationary Poisson hyperplane process with respect to $L$ (thus, a process of $(k-1)$ )-flats in $L$ ), which is denoted by $X \cap L$ (see [13, pp. 129ff.]). We denote the intensity of this section process by $\gamma_{L}$ and its directional distribution, defined on $\mathbb{S}_{L}$, by $\varphi_{L}$. These data depend in the following way on $\gamma$ and $\varphi$. Let $(\cdot) \mid L$ denote the orthogonal projection to $L$. The spherical projection of the measure $\varphi$ to $\mathbb{S}_{L}$ is defined by

$$
\left(\pi_{L} \varphi\right)(A):=\int_{\mathbb{S}^{d-1} \backslash L^{\perp}} \mathbf{1}_{A}\left(\frac{\boldsymbol{u} \mid L}{\|\boldsymbol{u} \mid L\|}\right)\|\boldsymbol{u} \mid L\| \varphi(\mathrm{d} \boldsymbol{u})
$$

for $A \in \mathscr{B}\left(\mathbb{S}_{L}\right)$. Then

$$
\gamma_{L} \varphi_{L}=\gamma \pi_{L} \varphi
$$

This is Equation (3.2) of [4] and a special case of [13, Theorem 4.4.7]. In particular,

$$
\gamma_{L}=\gamma \int_{\mathbb{S}^{d-1} \backslash L^{\perp}}\|\boldsymbol{u} \mid L\| \varphi(\mathrm{d} \boldsymbol{u})
$$

Note that $\gamma_{L}$ depends also on $\varphi$, although this is not shown by the notation. The measure $\varphi_{L}$ depends only on $\varphi$ and $L$.

Finally, we need two useful auxiliary convex bodies. The associated zonoid $\Pi_{X}$ of $X$ is the $\boldsymbol{o}$-symmetric convex body with support function given by

$$
h\left(\Pi_{X}, \boldsymbol{u}\right)=\frac{\gamma}{2} \int_{\mathbb{S}^{d-1}}|\langle\boldsymbol{u}, \boldsymbol{v}\rangle| \varphi(\mathrm{d} \boldsymbol{v}), \quad \boldsymbol{u} \in \mathbb{S}^{d-1} .
$$

The Blaschke body $B_{X}$ of $X$ is the $\boldsymbol{o}$-symmetric convex body with surface area measure

$$
S_{d-1}\left(B_{X}, \cdot\right)=\gamma \varphi .
$$

For the notion of the surface area measure, see [13, p. 607] or Section 4.2 of [11]. The existence and uniqueness of the Blaschke body follow from Minkowski's theorem (see, e.g. [11, Theorem 7.1.2]).

For a subspace $L \in G(d, k)$, we denote the associated zonoid of the section process $X \cap L$ by $\Pi_{X \cap L}$ and its Blaschke body by $B_{X \cap L}$. Both are convex bodies in $L$. We have

$$
\Pi_{X \cap L}=\Pi_{X} \mid L
$$

(due to Matheron; see [13, Equation (4.61)]). According to (3), the surface area measure $S_{k-1}^{L}\left(B_{X \cap L}, \cdot\right)$ of $B_{X \cap L}$ with $L$ as ambient space is given by

$$
S_{k-1}^{L}\left(B_{X \cap L}, \cdot\right)=\gamma \pi_{L} \varphi ;
$$

this is a measure on the unit sphere $\mathbb{S}_{L}$ in $L$. 


\section{Typical faces with given directions}

The direction $D(K) \in G(d, k)$ of a $k$-dimensional convex body $K \subset \mathbb{R}^{d}$ is the linear subspace which is a translate of the affine hull of $K$; it can also be written as $D(K)=\operatorname{lin}(K-K)$, the linear hull of the vector sum of $K$ and $-K$. The mapping $D$ from the space of $k$-dimensional convex bodies to $G(d, k)$ is continuous.

The random polytope $Z_{0}^{(k)}$ takes its values in $\mathcal{K}$, which is a Polish space. Therefore, the regular conditional distribution of $Z_{0}^{(k)}$, given the direction $D\left(Z_{0}^{(k)}\right)=L$, exists for $L \in$ $G(d, k)$. We denote it by $\mathrm{P}\left\{Z_{0}^{(k)} \in \cdot \mid D\left(Z_{0}^{(k)}\right)=L\right\}$. Similarly, $\mathrm{P}\left\{Z^{(k)} \in \cdot \mid D\left(Z^{(k)}\right)=L\right\}$ denotes the regular conditional distribution of the typical $k$-face $Z^{(k)}$, given the direction $D\left(Z^{(k)}\right)=L$, for $L \in G(d, k)$.

The following theorem describes these conditional distributions. As a by-product, it also provides the directional distributions of the weighted typical $k$-face and of the typical $k$-face, that is, the distributions of $D\left(Z_{0}^{(k)}\right)$ and $D\left(Z^{(k)}\right)$.

Theorem 1. Let $k \in\{1, \ldots, d-1\}$. The directional distribution of the weighted typical $k$-face is equal to $\mathrm{Q}_{d-k}$.

For $\mathrm{Q}_{d-k}$-almost all $L \in G(d, k)$, the conditional distribution of the weighted typical $k$-face $Z_{0}^{(k)}$, given the direction $D\left(Z_{0}^{(k)}\right)=L$, is equal to the distribution of the zero cell of a stationary Poisson hyperplane process in $L$ with intensity $\gamma_{L}$ and spherical directional distribution $\varphi_{L}$.

The directional distribution $R_{k}$ of the typical $k$-face is given by

$$
R_{k}(C)=\frac{V_{d-k}\left(\Pi_{X}\right)}{\left(\begin{array}{l}
d \\
k
\end{array}\right) V_{d}\left(\Pi_{X}\right)} \int_{C} V_{k}\left(\Pi_{X} \mid L\right) \mathrm{Q}_{d-k}(\mathrm{~d} L)
$$

for $C \in \mathscr{B}(G(d, k))$ (and hence is equivalent to $\mathrm{Q}_{d-k}$ ).

For $\mathrm{Q}_{d-k}$-almost all $L \in G(d, k)$, the conditional distribution of the typical $k$-face $Z^{(k)}$, given that $D\left(Z^{(k)}\right)=L$, is equal to the distribution of the typical cell of a stationary Poisson hyperplane process in $L$ with intensity $\gamma_{L}$ and spherical directional distribution $\varphi_{L}$.

Proof. A crucial tool is the following integral representation for the distribution of the weighted typical $k$-face $Z_{0}^{(k)}$, which was obtained in [12, Theorem 1]. For $A \in \mathcal{B}(\mathcal{K})$,

$$
\mathrm{P}\left\{Z_{0}^{(k)} \in A\right\}=\int_{G(d, k)} \mathrm{P}\left\{Z_{0} \cap L \in A\right\} \mathrm{Q}_{d-k}(\mathrm{~d} L) .
$$

For $C \in \mathscr{B}(G(d, k))$, we obtain, since $D\left(Z_{0} \cap L\right)=L$ almost surely,

$$
\mathrm{P}\left\{Z_{0}^{(k)} \in A, D\left(Z_{0}^{(k)}\right) \in C\right\}=\int_{C} \mathrm{P}\left\{Z_{0} \cap L \in A\right\} \mathrm{Q}_{d-k}(\mathrm{~d} L) ;
$$

in particular, $\mathrm{P}\left\{D\left(Z_{0}^{(k)}\right) \in C\right\}=\mathrm{Q}_{d-k}(C)$. Thus, $\mathrm{Q}_{d-k}$ is equal to the distribution $\mathrm{P}_{D\left(Z_{0}^{(k)}\right)}$ of $D\left(Z_{0}^{(k)}\right)$ (this can also be seen in a more direct way).

By the definition of the conditional distribution,

$$
\mathrm{P}\left\{Z_{0}^{(k)} \in A, D\left(Z_{0}^{(k)}\right) \in C\right\}=\int_{C} \mathrm{P}\left\{Z_{0}^{(k)} \in A \mid D\left(Z_{0}^{(k)}\right)=L\right\} \mathrm{P}_{D\left(Z_{0}^{(k)}\right)}(\mathrm{d} L) .
$$

Since this equation and (7) hold for all $C \in \mathscr{B}(G(d, k))$, we deduce that

$$
\mathrm{P}\left\{Z_{0}^{(k)} \in A \mid D\left(Z_{0}^{(k)}\right)=L\right\}=\mathrm{P}\left\{Z_{0} \cap L \in A\right\} \text { for } \mathrm{Q}_{d-k} \text {-almost all } L \in G(d, k) .
$$


Here the exceptional set depends on $A$, but since the $\sigma$-algebra $\mathcal{B}(\mathcal{K})$ is countably generated, there is a $\mathrm{Q}_{d-k}$ null set $N \subset G(d, k)$ such that

$$
\mathrm{P}\left\{Z_{0}^{(k)} \in \cdot \mid D\left(Z_{0}^{(k)}\right)=L\right\}=\mathrm{P}\left\{Z_{0} \cap L \in \cdot\right\}
$$

for all $L \in G(d, k) \backslash N$.

It remains to observe that $Z_{0} \cap L=Z_{0}(X \cap L)$, where $Z_{0}(X \cap L)$ denotes the zero cell of the section process $X \cap L$, and that $X \cap L$ is a stationary Poisson hyperplane process in $L$ with intensity $\gamma_{L}$ and spherical directional distribution $\varphi_{L}$. This proves the part of Theorem 1 that refers to the weighted typical $k$-face.

For the second part, concerning the typical $k$-face, let $h$ be a translation invariant, nonnegative, measurable function on the space of $k$-dimensional polytopes, and set $f(K):=$ $h(K) V_{k}(K)^{-1}$ for such polytopes $K$. Then (2) gives

$$
\mathrm{E} h\left(Z^{(k)}\right)=\mathrm{E} V_{k}\left(Z^{(k)}\right) \mathrm{E}\left[h\left(Z_{0}^{(k)}\right) V_{k}\left(Z_{0}^{(k)}\right)^{-1}\right] .
$$

We apply (6), observe that $Z_{0} \cap L=Z_{0}(X \cap L)$, then apply (2) again, this time in $L$, where we denote by $Z(X \cap L)$ the typical cell of $X \cap L$. This yields

$$
\begin{aligned}
\mathrm{E} h\left(Z^{(k)}\right) & =\mathrm{E} V_{k}\left(Z^{(k)}\right) \int_{G(d, k)} \mathrm{E}\left[h\left(Z_{0}(X \cap L)\right) V_{k}\left(Z_{0}(X \cap L)\right)^{-1}\right] \mathrm{Q}_{d-k}(\mathrm{~d} L) \\
& =\mathrm{E} V_{k}\left(Z^{(k)}\right) \int_{G(d, k)} \frac{1}{\mathrm{E} V_{k}(Z(X \cap L))} \mathrm{E} h(Z(X \cap L)) \mathrm{Q}_{d-k}(\mathrm{~d} L) \\
& =\int_{G(d, k)} \mathrm{E} h(Z(X \cap L)) R_{k}(\mathrm{~d} L)
\end{aligned}
$$

with

$$
R_{k}(C)=\int_{C} \frac{\mathrm{E} V_{k}\left(Z^{(k)}\right)}{\mathrm{E} V_{k}(Z(X \cap L))} \mathrm{Q}_{d-k}(\mathrm{~d} L)
$$

for $C \in \mathscr{B}(G(d, k))$. From [13, Equations (10.43), (10.3), (10.44)] we know that

$$
\mathrm{E} V_{k}\left(Z^{(k)}\right)=\frac{V_{d-k}\left(\Pi_{X}\right)}{\left(\begin{array}{l}
d \\
k
\end{array}\right) V_{d}\left(\Pi_{X}\right)} .
$$

This can be applied to $X \cap L$, with $\mathbb{R}^{d}$ replaced by the $k$-dimensional space $L$, and together with (4) this gives

and, thus, (5).

$$
\mathrm{E} V_{k}(Z(X \cap L))=\frac{1}{V_{k}\left(\Pi_{X \cap L}\right)}=\frac{1}{V_{k}\left(\Pi_{X} \mid L\right)}
$$

Let $A \in \mathscr{B}(\mathcal{K})$. We apply the result (8) to the function defined by $h(K)=\mathbf{1}_{A}(K-s(K))$, where $s(K)$ denotes the Steiner point of $K$. Since our notion of typical faces and cells uses the Steiner point as the centre function, we have $s\left(Z^{(k)}\right)=\boldsymbol{o}$ and $s(Z(X \cap L))=\boldsymbol{o}$. Thus, we arrive at

$$
\mathrm{P}\left\{Z^{(k)} \in A\right\}=\int_{G(d, k)} \mathrm{P}\{Z(X \cap L) \in A\} R_{k}(\mathrm{~d} L) .
$$

This equation describes the distribution of the typical $k$-face of the stationary Poisson hyperplane mosaic $X^{(d)}$ in terms of typical cells in $k$-dimensional subspaces and is thus a counterpart to Theorem 1 of [12]. It can be employed similarly as (6) was used above. In this way, the proof of Theorem 1 is completed. 


\section{Comparison with given shapes}

The typical $k$-face $Z^{(k)}$ and weighted typical $k$-face $Z_{0}^{(k)}$ are random polytopes, and we want to study their 'preferred' shapes under different conditions. The question for a 'mean shape' can be approached in different ways. If $K$ is a random convex body in $\mathbb{R}^{d}$ satisfying a suitable integrability condition, we can define its Aumann expectation, which is closely related to Minkowski addition. In our context, a different type of mean body, related to Blaschke addition, is more appropriate. The Blaschke sum $K \# M$ of two $d$-dimensional convex bodies $K$ and $M$ is the unique convex body with surface area measure $S_{d-1}(K \# M, \cdot)=S_{d-1}(K, \cdot)+S_{d-1}(M, \cdot)$ and, say, Steiner point at $\boldsymbol{o}$. Let $C$ be a $d$-dimensional random convex body in $\mathbb{R}^{d}$ with $\mathrm{E} V_{d-1}(C)<\infty$. Then the Blaschke expectation (see [9, p. 200]) of $C$ can be defined as the convex body $\mathrm{E}_{\mathrm{BL}}(C)$ with $S_{d-1}\left(\mathrm{E}_{\mathrm{BL}}(C), \cdot\right)=\mathrm{E} S_{d-1}(C, \cdot)$ and Steiner point at $\boldsymbol{o}$. As indicated by Molchanov (loc. cit.), it is possible to derive a strong law of large numbers for normalized Blaschke sums.

For the typical cell $Z$, which is the typical grain of the stationary particle process $X^{(d)}$, it turns out that its Blaschke expectation is a dilate of the Blaschke body $B_{X}$ of the hyperplane process $X$ (this follows from [13, pp. 488-489]). This is one instance of the existing relations between the shape of the typical cell and the Blaschke body of $X$. Another one is expressed in the following theorem. Recall that a homothet of a convex body $K \subset \mathbb{R}^{d}$ is any body of the form $\lambda K+t$ with $\lambda>0$ and $t \in \mathbb{R}^{d}$.

Theorem 2. Among all convex bodies $C$ of given volume $V_{d}(C)>0$, precisely the homothets of the Blaschke body $B_{X}$ maximize the probability that the typical cell $Z$ contains a translate of $C$.

A similar result holds for the zero cell, but without admitting translations (see [13, Theorem 10.4.11]). Theorem 2 requires a different approach. We extend the proof of [13, Theorem 10.4.8], which uses an idea of Miles [8] to determine the distribution of the inradius of the typical cell; we generalize this from the usual inradius to the relative inradius. Let $C$ be a convex body of positive dimension. For a convex body $K$ containing some positive homothet of $C$, the relative inradius of $K$ with respect to $C$ is defined by

$$
r_{C}(K):=\max \left\{\lambda>0 \text { : there exists } z \in \mathbb{R}^{d} \text { with } \lambda C+z \subset K\right\} .
$$

We shall show that

$$
\mathrm{P}\left\{r_{C}(Z)>a\right\}=\mathrm{e}^{-2 d V\left(C, B_{X}, \ldots, B_{X}\right) a} \quad \text { for } a \geq 0,
$$

where $V$ denotes the mixed volume. From (9) we can then deduce that

$$
\begin{aligned}
\mathrm{P}\{Z \text { contains a translate of } C\} & =\mathrm{P}\left\{r_{C}(Z) \geq 1\right\} \\
& =\mathrm{e}^{-2 d V\left(C, B_{X}, \ldots, B_{X}\right)} \\
& \leq \exp \left[-2 d V_{d}(C)^{1 / d} V_{d}\left(B_{X}\right)^{1-1 / d}\right],
\end{aligned}
$$

by Minkowski's inequality (see, e.g. [11, Theorem 6.2.1]). Equality holds if and only if $C$ is homothetic to the Blaschke body $B_{X}$. This is the assertion of Theorem 2 .

We remark that assertion (9) holds whenever $C$ has positive dimension. We prove it under this assumption. 
Proof of (9). We modify the proof of [13, Theorem 10.4.8] as far as necessary. For a convex body $K$, let

$$
\mathscr{H}_{K}=\{H \in A(d, d-1): H \cap K \neq \varnothing\} .
$$

We assume, without loss of generality, that $\boldsymbol{o} \in C$. Let $a \geq 0$, and set

$$
\Omega_{a}:=\left\{\omega \in \Omega: X(\omega)\left(\mathscr{H}_{a C}\right)=0\right\} .
$$

Every hyperplane $H \in A(d, d-1) \backslash \mathscr{H}_{a C}$ is of the form $H=H(\boldsymbol{u}, t)$ with $\boldsymbol{u} \in \mathbb{S}^{d-1}$ and $t>h(a C, \boldsymbol{u})$, where $h$ denotes the support function. We define a map $T_{a}: A(d, d-1) \backslash \mathscr{H}_{a C} \rightarrow$ $A(d, d-1)$ by $T_{a} H(\boldsymbol{u}, t):=H(\boldsymbol{u}, t-h(a C, \boldsymbol{u}))$. Let $\mathrm{A}_{a}$ be the trace $\sigma$-algebra of $\mathrm{A}$ on $\Omega_{a}$, and let $\mathrm{P}_{a}$ be the restriction of $\mathrm{P} / \mathrm{P}\left(\Omega_{a}\right)$ to $\Omega_{a}$. On the probability space $\left(\Omega_{a}, \mathrm{~A}_{a}, \mathrm{P}_{a}\right)$ we define a simple hyperplane process $X_{a}$ by $X_{a}(\omega):=\left\{T_{a} H: H \in X(\omega)\right\}, \omega \in \Omega_{a}$.

Let $A \in \mathscr{B}(A(d, d-1)$ ) (with $\boldsymbol{O} \notin H$ for all $H \in A$, without loss of generality). We have

$$
\begin{aligned}
\Theta\left(T_{a}^{-1}(A)\right) & =2 \gamma \int_{\mathbb{S}^{d-1}} \int_{h(a C, \boldsymbol{u})}^{\infty} \mathbf{1}_{T_{a}^{-1}(A)}(H(\boldsymbol{u}, t)) \mathrm{d} t \varphi(\mathrm{d} \boldsymbol{u}) \\
& =2 \gamma \int_{\mathbb{S}^{d-1}} \int_{h(a C, \boldsymbol{u})}^{\infty} \mathbf{1}_{A}(H(\boldsymbol{u}, t-h(a C, \boldsymbol{u}))) \mathrm{d} t \varphi(\mathrm{d} \boldsymbol{u}) \\
& =2 \gamma \int_{\mathbb{S}^{d-1}} \int_{0}^{\infty} \mathbf{1}_{A}(H(\boldsymbol{u}, t)) \mathrm{d} t \varphi(\mathrm{d} \boldsymbol{u}) \\
& =\Theta(A) .
\end{aligned}
$$

Let $k \in \mathbb{N}_{0}$. Since $T_{a}^{-1}(A) \cap \mathscr{H}_{a C}=\varnothing$, the independence property of Poisson processes gives

$$
\begin{aligned}
\mathrm{P}_{a}\left\{X_{a}(A)=k\right\} & =\mathrm{P}\left(\Omega_{a}\right)^{-1} \mathrm{P}\left\{X\left(T_{a}^{-1}(A)\right)=k, X\left(\mathscr{H}_{a C}\right)=0\right\} \\
& =\mathrm{P}\left\{X\left(T_{a}^{-1}(A)\right)=k\right\} \\
& =\mathrm{e}^{-\Theta\left(T_{a}^{-1}(A)\right)} \frac{\Theta\left(T_{a}^{-1}(A)\right)^{k}}{k !} \\
& =\mathrm{e}^{-\Theta(A)} \frac{\Theta(A)^{k}}{k !} \\
& =\mathrm{P}\{X(A)=k\} .
\end{aligned}
$$

It follows (e.g. from [13, Theorem 3.1.1]) that $X_{a}$ and $X$ are equal in distribution.

With every hyperplane $H \in X$ we associate the slab $H_{a}:=H-a C$. The cells induced by $X$ and $a$ are defined as the closures of the connected components of $\mathbb{R}^{d} \backslash \bigcup_{H \in X} H_{a}$. The system $X^{(d, a)}$ of all these cells is a stationary particle process. We denote its intensity by $\gamma^{(d, a)}$ and its grain distribution by $\mathrm{Q}^{(d, a)}$. If $\omega \in \Omega_{a}$ then among the cells induced by $X(\omega)$ and $a$ there is one containing $\boldsymbol{o}$; we denote it by $Z_{0}^{(a)}$. This defines a random polytope on $\left(\Omega_{a}, \mathrm{~A}_{a}, \mathrm{P}_{a}\right)$; it is equal to the zero cell of the hyperplane mosaic induced by $X_{a}$. Since $X_{a}$ and $X$ are stochastically equivalent, $Z_{0}^{(a)}$ and $Z_{0}$ are also stochastically equivalent. Now we can exactly copy the argument in $\left[13\right.$, p. 503], with $\mathrm{e}^{-2 \widehat{\gamma} a}$ replaced by $\mathrm{P}\left(\Omega_{a}\right)$, to derive

$$
\gamma^{(d, a)}=\mathrm{P}\left(\Omega_{a}\right) \gamma^{(d, 0)} .
$$

Let $K$ be a $d$-dimensional convex body, and suppose that $\lambda C+\boldsymbol{x} \subset K$, where $\lambda$ is maximal. Then $\boldsymbol{x}$ is called a relative incentre of $K$. The set $\mathcal{Z}(K)$ of all relative incentres of $K$ is a convex 
body (often one-pointed, but not always), and we denote by $z(K)$ its Steiner point. The function $z$ is translation covariant and measurable, and, hence, can be used as a centre function (in the sense of [13, Section 4.1]). We do this, and write $\mathcal{K}_{0}:=\{K \in \mathcal{K}: z(K)=\boldsymbol{o}\}$. (Since the relative inradius is translation invariant, the distribution of the relative inradius of the typical cell does not depend on the choice of the centre function.)

Now we observe that to every cell $K \in X^{(d)}$ with $r_{C}(K)>a$ there corresponds a unique cell $K_{a} \in X^{(d, a)}$ contained in it: if

$$
K=\bigcap_{i=1}^{m}\left\{\boldsymbol{x}:\left\langle\boldsymbol{x}, \boldsymbol{u}_{i}\right\rangle \leq t_{i}\right\}
$$

then

$$
K_{a}=\bigcap_{i=1}^{m}\left\{\boldsymbol{x}:\left\langle\boldsymbol{x}, \boldsymbol{u}_{i}\right\rangle \leq t_{i}-\operatorname{ah}\left(C, \boldsymbol{u}_{i}\right)\right\} .
$$

If $r_{C}(K)=\lambda$, the set of relative incentres of $K$ is given by $\mathcal{Z}(K)=K \sim \lambda C$, where ' $~ '$ denotes the Minkowski difference (see [11, p. 133]). We have $K_{a}=K \sim a C$ and, hence (by [11, Equation (3.3.14)]), $K_{a} \sim(\lambda-a) C=K \sim \lambda C \neq \varnothing$. This shows that $r_{C}\left(K_{a}\right) \geq \lambda-a$, and, from $K_{a}+a C \subset K$, it follows that $r_{C}\left(K_{a}\right)+a \leq r_{C}(K)=\lambda$; hence, $r_{C}\left(K_{a}\right)=\lambda-a$. Therefore, $\mathcal{Z}\left(K_{a}\right)=K_{a} \sim(\lambda-a) C=\mathcal{Z}(K)$. We conclude that $z\left(K_{a}\right)=\boldsymbol{z}(K)$.

Let $B \in \mathscr{B}\left(\mathbb{R}^{d}\right)$ be a set with Lebesgue measure 1 . Since $\mathrm{Q}^{(d, 0)}$ is the distribution of the typical cell $Z$, we obtain, from [13, Theorem 4.1.3(a)],

$$
\begin{aligned}
\mathrm{P}\left\{r_{C}(Z)>a\right\} & =\int_{\mathcal{K}_{0}} \mathbf{1}_{(a, \infty)}\left(r_{C}(K)\right) \mathrm{Q}^{(d, 0)}(\mathrm{d} K) \\
& =\frac{1}{\gamma^{(d, 0)}} \mathrm{E} \sum_{K \in X^{(d)}, z(K) \in B} \mathbf{1}_{(a, \infty)}\left(r_{C}(K)\right) \\
& =\frac{1}{\gamma^{(d, 0)}} \mathrm{E} \sum_{K_{a} \in X^{(d, a)}, z\left(K_{a}\right) \in B} 1 \\
& =\frac{1}{\gamma^{(d, 0)}} \gamma^{(d, a)} \\
& =\mathrm{P}\left(\Omega_{a}\right)
\end{aligned}
$$

by (10). It remains to remark that, by the Poisson property, $\mathrm{P}\left(\Omega_{a}\right)=\mathrm{e}^{-\Theta\left(\mathscr{H}_{a C}\right)}$, and (1) gives

$$
\begin{aligned}
\Theta\left(\mathscr{H}_{a C}\right) & =2 \gamma \int_{\mathbb{S}^{d-1}} h(a C, \boldsymbol{u}) \varphi(\mathrm{d} \boldsymbol{u}) \\
& =2 a \int_{\mathbb{S}^{d-1}} h(C, \boldsymbol{u}) S_{d-1}\left(B_{X}, \mathrm{~d} \boldsymbol{u}\right) \\
& =2 d V\left(C, B_{X}, \ldots, B_{X}\right) a
\end{aligned}
$$

where a formula for mixed volumes ([13, Equation (14.23)]) was used. This yields

$$
\mathrm{P}\left(\Omega_{a}\right)=\mathrm{e}^{-2 d V\left(C, B_{X}, \ldots, B_{X}\right) a},
$$

completing the proof of (9). 
We can now use Theorem 1 to derive from Theorem 2 and [13, Theorem 10.4.11] the following result.

Theorem 3. Let $k \in\{1, \ldots, d-1\}$. For each $L \in G(d, k)$, let $C_{L} \subset L$ be a convex body with $V_{k}\left(C_{L}\right)=1$. Then, for $\mathrm{Q}_{d-k}$-almost all $L \in G(d, k)$, the following holds.

The conditional probability that the typical $k$-face $Z^{(k)}$ contains a translate of $C_{L}$, given that $D\left(Z^{(k)}\right)=L$, is at most

$$
\exp \left[-2 k V_{k}\left(B_{X \cap L}\right)^{1-1 / k}\right]
$$

and it is equal to this value if and only if $C_{L}$ is homothetic to $B_{X \cap L}$.

Assume in addition that $\boldsymbol{o} \in C_{L}$ for $L \in G(d, k)$. The conditional probability that the weighted typical $k$-face $Z_{0}^{(k)}$ contains $C_{L}$, given that $D\left(Z^{(k)}\right)=L$, is at most the value (11), with equality if and only if $C_{L}$ is homothetic to $B_{X \cap L}$.

\section{Large faces}

The solution of D. G. Kendall's problem for the zero cell of anisotropic stationary Poisson hyperplane processes, mentioned in the introduction, was extended in [4] to weighted typical $k$-faces with large $k$-dimensional volume and direction in a small neighbourhood of a given direction. In this section, we state a version of this result for the conditional distribution of a weighted typical $k$-face, given its direction and given that it has large $k$-volume.

To define the conditional probabilities in question, we consider, for any $a \geq 1$, the random variable $\eta_{a}: \Omega \rightarrow\{0,1\}$ defined by $\eta_{a}:=\mathbf{1}\left\{V_{k}\left(Z_{0}^{(k)}\right) \geq a\right\}$, where $\{0,1\}$ is endowed with the $\sigma$-algebra generated by the singletons. We denote by

$$
\mathrm{P}\left\{Z_{0}^{(k)} \in \cdot \mid\left(\eta_{a}, D\left(Z_{0}^{(k)}\right)\right)=(b, L)\right\}
$$

the regular conditional probability distribution of $Z_{0}^{(k)}$, given that $\left(\eta_{a}, D\left(Z_{0}^{(k)}\right)\right)=(b, L)$ for $(b, L) \in\{0,1\} \times G(d, k)$.

Lemma 1. A version of the regular conditional probability distribution (12) is given by

$$
\begin{aligned}
\varrho_{(b, L)}(\cdot):= & \frac{\mathrm{P}\left\{Z_{0}(X \cap L) \in \cdot, V_{k}\left(Z_{0}(X \cap L)\right)<a\right\}}{\mathrm{P}\left\{V_{k}\left(Z_{0}(X \cap L)\right)<a\right\}} \mathbf{1}\{b=0\} \\
& +\frac{\mathrm{P}\left\{Z_{0}(X \cap L) \in \cdot, V_{k}\left(Z_{0}(X \cap L)\right) \geq a\right\}}{\mathrm{P}\left\{V_{k}\left(Z_{0}(X \cap L)\right) \geq a\right\}} \mathbf{1}\{b=1\}
\end{aligned}
$$

for $(b, L) \in\{0,1\} \times G(d, k)$.

Proof. For $L \in G(d, k)$, we define a measure $\mu_{L}$ on $\{0,1\}$ by

$$
\mu_{L}:=\mathrm{P}\left\{V_{k}\left(Z_{0}(X \cap L)\right)<a\right\} \delta_{0}+\mathrm{P}\left\{V_{k}\left(Z_{0}(X \cap L)\right) \geq a\right\} \delta_{1},
$$

where $\delta_{0}$ and $\delta_{1}$ are the Dirac measures at 0 and 1 , respectively. Then (7) implies that

$$
\mathrm{P}\left\{\left(\eta_{a}, D\left(Z_{0}^{(k)}\right)\right) \in \cdot\right\}=\int_{G(d, k)} \int_{\{0,1\}} \mathbf{1}\{(b, L) \in \cdot\} \mu_{L}(\mathrm{~d} b) \mathrm{Q}_{d-k}(\mathrm{~d} L) .
$$


For Borel sets $A \subset \mathcal{K}$ and $C \subset G(d, k)$, and for $B \subset\{0,1\}$, we obtain

$$
\begin{aligned}
& \int_{\{0,1\} \times G(d, k)} \varrho_{(b, L)}(A) \mathbf{1}\{(b, L) \in B \times C\} \mathrm{P}_{\left(\eta_{a}, D\left(Z_{0}^{(k)}\right)\right)}(\mathrm{d}(b, L)) \\
& =\int_{C} \int_{B} \varrho_{(b, L)}(A) \mu_{L}(\mathrm{~d} b) \mathrm{Q}_{d-k}(\mathrm{~d} L) \\
& =\int_{C}\left[\mathrm{P}\left\{Z_{0}(X \cap L) \in A, V_{k}\left(Z_{0}(X \cap L)\right)<a\right\} \mathbf{1}_{B}(0)\right. \\
& \left.\quad+\mathrm{P}\left\{Z_{0}(X \cap L) \in A, V_{k}\left(Z_{0}(X \cap L)\right) \geq a\right\} \mathbf{1}_{B}(1)\right] \mathrm{Q}_{d-k}(\mathrm{~d} L) \\
& =\mathrm{P}\left\{Z_{0}^{(k)} \in A,\left(\eta_{a}, D\left(Z_{0}^{(k)}\right)\right) \in B \times C\right\},
\end{aligned}
$$

from which the assertion follows.

In the following, we choose $\varrho_{(b, L)}(\cdot)$ as a definite version of the regular conditional probability distribution (12). Thus, introducing the notation

$$
\mathrm{P}\left\{Z_{0}^{(k)} \in \cdot \mid V_{k}\left(Z_{0}^{(k)}\right) \geq a, D\left(Z_{0}^{(k)}\right)=L\right\}:=\mathrm{P}\left\{Z_{0}^{(k)} \in \cdot \mid\left(\eta_{a}, D\left(Z_{0}^{(k)}\right)\right)=(1, L)\right\}
$$

for $a \geq 1$ and $L \in G(d, k)$, we obtain

$$
\mathrm{P}\left\{Z_{0}^{(k)} \in \cdot \mid V_{k}\left(Z_{0}^{(k)}\right) \geq a, D\left(Z_{0}^{(k)}\right)=L\right\}=\frac{\mathrm{P}\left\{Z_{0}(X \cap L) \in \cdot, V_{k}\left(Z_{0}(X \cap L)\right) \geq a\right\}}{\mathrm{P}\left\{V_{k}\left(Z_{0}(X \cap L)\right) \geq a\right\}} .
$$

For $L \in G(d, k)$, in addition to the Blaschke body $B_{X \cap L}$ of the section process $X \cap L$, we introduce the scaled version $B_{L} \subset L$, which is defined by $S_{k-1}^{L}\left(B_{L}, \cdot\right)=\varphi_{L}$. Moreover, for a $k$-dimensional convex body $K \subset L$, we measure the deviation (in shape) of $K$ from $B_{L}$ by the function

$$
\vartheta\left(K, B_{L}\right)=\log \min \left\{\frac{\beta}{\alpha}: \alpha, \beta>0, \text { there exists } z \in L: \alpha B_{L} \subset K+z \subset \beta B_{L}\right\} .
$$

The constants $c_{1}, c_{2}, \ldots$ appearing in the following may depend on $\varphi, \gamma$, and $\varepsilon$; the precise dependence will be indicated in each case.

By Lemma 4.2 of [4] (recall that $Z_{0} \cap L=Z_{0}(X \cap L)$ ), the following is true. For $0<\varepsilon<1$, there exists a constant $c_{1}>0$, depending only on $\varphi, \gamma$, and $\varepsilon$, and a constant $c_{2}>0$, depending only on $\varphi$, such that, for $L \in G(d, k), a \geq 1$, and $h \in\left(0, \frac{1}{2}\right)$,

$$
\begin{aligned}
& \mathrm{P}\left\{\vartheta\left(Z_{0}(X \cap L), B_{L}\right) \geq \varepsilon, V_{k}\left(Z_{0}(X \cap L)\right) \in a(1,1+h)\right\} \\
& \quad \leq c_{1} h \exp \left[-2\left(1+2 c_{2} \varepsilon^{k+1}\right) \gamma_{L} \tau_{L} a^{1 / k}\right]
\end{aligned}
$$

where $\tau_{L}=k V_{k}\left(B_{L}\right)^{1-1 / k}$.

By Lemma 4.1 of [4], there exist constants $c_{3}$ and $0<h_{0}<\frac{1}{2}$, depending only on $\varphi, \gamma$, and $\varepsilon$, such that

$$
\begin{aligned}
\mathrm{P}\left\{V_{k}\left(Z_{0}(X \cap L)\right) \geq a\right\} & \geq \mathrm{P}\left\{V_{k}\left(Z_{0}(X \cap L)\right) \in a\left(1,1+h_{0}\right)\right\} \\
& \geq c_{3} h_{0} \exp \left[-2\left(1+\left(\frac{c_{2}}{2}\right) \varepsilon^{k+1}\right) \gamma_{L} \tau_{L} a^{1 / k}\right] .
\end{aligned}
$$


We apply (14) with $h=h_{0}$. Since $L \mapsto \gamma_{L} \tau_{L}$ is continuous and therefore bounded from below by a positive constant depending only on $\varphi$ and $\gamma$, we obtain as in [5, pp. 1164-1165, Case 2],

$$
\begin{gathered}
\mathrm{P}\left\{\vartheta\left(Z_{0}(X \cap L), B_{L}\right) \geq \varepsilon, V_{k}\left(Z_{0}(X \cap L)\right) \geq a\right\} \\
\leq c_{4} h_{0} \exp \left[-2\left(1+c_{2} \varepsilon^{k+1}\right) \gamma_{L} \tau_{L} a^{1 / k}\right],
\end{gathered}
$$

where $c_{4}>0$ is a constant which depends only on $\varphi, \gamma$, and $\varepsilon$. Hence, for $L \in G(d, k)$ and $a \geq 1$, we obtain

$$
\mathrm{P}\left\{\vartheta\left(Z_{0}^{(k)}, B_{L}\right) \geq \varepsilon \mid V_{k}\left(Z_{0}^{(k)}\right) \geq a, D\left(Z_{0}^{(k)}\right)=L\right\} \leq c_{5} \exp \left[-c_{2} \varepsilon^{k+1} \gamma_{L} \tau_{L} a^{1 / k}\right],
$$

where $c_{5}>0$ is a constant which depends only on $\varphi, \gamma$, and $\varepsilon$. Thus, we have proved the following theorem.

Theorem 4. Let $\varepsilon \in(0,1)$ and $a \geq 1$. There exist constants $c_{6}, c_{7}>0$, where $c_{6}$ depends only on $\varphi, \gamma$, and $\varepsilon$, and $c_{7}$ depends only on $\varphi$ and $\gamma$, such that

$$
\mathrm{P}\left\{\vartheta\left(Z_{0}^{(k)}, B_{L}\right) \geq \varepsilon \mid V_{k}\left(Z_{0}^{(k)}\right) \geq a, D\left(Z_{0}^{(k)}\right)=L\right\} \leq c_{6} \exp \left[-c_{7} \varepsilon^{k+1} a^{1 / k}\right]
$$

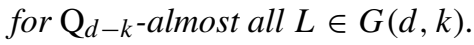

We note that, for the special version given by (13), the estimate of Theorem 4 is valid for all $L \in G(d, k)$.

Now we denote by $\mathrm{P}\left\{Z_{0}^{(k)} \in \cdot \mid\left(V_{k}\left(Z_{0}^{(k)}\right), D\left(Z_{0}^{(k)}\right)\right)=(a, L)\right\}$ the regular conditional probability distribution of $Z_{0}^{(k)}$, given that $\left(V_{k}\left(Z_{0}^{(k)}\right), D\left(Z_{0}^{(k)}\right)\right)=(a, L)$. A similar argument as above leads to an estimate for

$$
\mathrm{P}\left\{\vartheta\left(Z_{0}^{(k)}, B_{L}\right) \geq \varepsilon \mid V_{k}\left(Z_{0}^{(k)}\right)=a, D\left(Z_{0}^{(k)}\right)=L\right\} .
$$

To show this, we first remark that (7) implies that

$$
\mathrm{P}\left\{\left(V_{k}\left(Z_{0}^{(k)}\right), D\left(Z_{0}^{(k)}\right)\right) \in \cdot\right\}=\int_{G(d, k)} \int_{0}^{\infty} \mathbf{1}\{(t, L) \in \cdot\} \mathrm{P}_{V_{k}\left(Z_{0}(X \cap L)\right)}(\mathrm{d} t) \mathrm{Q}_{d-k}(\mathrm{~d} L) .
$$

Hence, for Borel sets $A \subset \mathcal{K}, C \subset G(d, k)$, and $I \subset[0, \infty)$, we deduce from (7) and (15) that

$$
\begin{aligned}
& \int_{C} \int_{I} \mathrm{P}\left\{Z_{0}^{(k)} \in A \mid\left(V_{k}\left(Z_{0}^{(k)}\right), D\left(Z_{0}^{(k)}\right)\right)=(t, L)\right\} \mathrm{P}_{V_{k}\left(Z_{0}(X \cap L)\right)}(\mathrm{d} t) \mathrm{Q}_{d-k}(\mathrm{~d} L) \\
& \quad=\int_{I \times C} \mathrm{P}\left\{Z_{0}^{(k)} \in A \mid\left(V_{k}\left(Z_{0}^{(k)}\right), D\left(Z_{0}^{(k)}\right)\right)=(t, L)\right\} \mathrm{P}_{\left(V_{k}\left(Z_{0}^{(k)}\right), D\left(Z_{0}^{(k)}\right)\right)}(\mathrm{d}(t, L)) \\
& \quad=\mathrm{P}\left\{Z_{0}^{(k)} \in A, V_{k}\left(Z_{0}^{(k)}\right) \in I, D\left(Z_{0}^{(k)}\right) \in C\right\} \\
& \quad=\int_{C} \mathrm{P}\left\{Z_{0}(X \cap L) \in A, V_{k}\left(Z_{0}(X \cap L)\right) \in I\right\} \mathrm{Q}_{d-k}(\mathrm{~d} L) \\
& =\int_{C} \int_{I} \mathrm{P}\left\{Z_{0}(X \cap L) \in A \mid V_{k}\left(Z_{0}(X \cap L)\right)=t\right\} \mathrm{P}_{V_{k}\left(Z_{0}(X \cap L)\right)}(\mathrm{d} t) \mathrm{Q}_{d-k}(\mathrm{~d} L),
\end{aligned}
$$

where $\mathrm{P}\left\{Z_{0}(X \cap L) \in \cdot \mid V_{k}\left(Z_{0}(X \cap L)\right)=t\right\}$ is the regular conditional probability distribution of $Z_{0}(X \cap L)$, given that $V_{k}\left(Z_{0}(X \cap L)\right)=t$. Therefore, for $\mathrm{P}_{\left(V_{k}\left(Z_{0}^{(k)}\right), D\left(Z_{0}^{(k)}\right)\right)}$-almost all $(t, L) \in(0, \infty) \times G(d, k)$,

$$
\mathrm{P}\left\{Z_{0}^{(k)} \in \cdot \mid\left(V_{k}\left(Z_{0}^{(k)}\right), D\left(Z_{0}^{(k)}\right)\right)=(t, L)\right\}=\mathrm{P}\left\{Z_{0}(X \cap L) \in \cdot \mid V_{k}\left(Z_{0}(X \cap L)\right)=t\right\} .
$$


Let $\lambda_{1}$ denote the Lebesgue measure on $\mathbb{R}$. Then, by the same arguments as in [2, Section 9], applied to the section process $X \cap L$ in $L$, we find that $\mathrm{P}\left\{V_{k}\left(Z_{0}(X \cap L)\right) \in \cdot\right\}$ and $\lambda_{1}$ have the same null sets and that, for a Borel set $A \subset \mathcal{K}, L \in G(d, k)$, and $\lambda_{1}$-almost all $t>0$,

$$
\begin{aligned}
& \mathrm{P}\left\{Z_{0}(X \cap L) \in A \mid V_{k}\left(Z_{0}(X \cap L)\right)=t\right\} \\
& \quad=\lim _{n \rightarrow \infty} \frac{\mathrm{P}\left\{Z_{0}(X \cap L) \in A, V_{k}\left(Z_{0}(X \cap L)\right) \in[t, t+1 / n]\right\}}{\mathrm{P}\left\{V_{k}\left(Z_{0}(X \cap L)\right) \in[t, t+1 / n]\right\}} .
\end{aligned}
$$

Lemma 4.2 of [4] shows that, for $\varepsilon \in(0,1)$ and $L \in G(d, k)$, there exist constants $c_{8}>0$, depending only on $\gamma, \varphi$, and $\varepsilon$, and $c_{9}>0$, depending only on $\gamma$ and $\varphi$, such that, for $t \geq 1$ and all integers $n \geq 3$,

$$
\begin{gathered}
\mathrm{P}\left\{\vartheta\left(Z_{0}(X \cap L), B_{L}\right) \geq \varepsilon, V_{k}\left(Z_{0}(X \cap L)\right) \in\left[t, t+\frac{1}{n}\right]\right\} \\
\leq \frac{c_{8}}{n t} \exp \left[-2\left(1+c_{9} \varepsilon^{k+1}\right) \gamma_{L} \tau_{L} t^{1 / k}\right] .
\end{gathered}
$$

In addition, Lemma 4.1 of [4] shows that, for $\varepsilon \in(0,1)$ and $L \in G(d, k)$, there exist constants $n_{0} \geq 3$ and $c_{10}$, depending only on $\gamma, \varphi$, and $\varepsilon$, such that, for $t \geq 1$ and all integers $n \geq n_{0}$,

$$
\mathrm{P}\left\{V_{k}\left(Z_{0}(X \cap L)\right) \in\left[t, t+\frac{1}{n}\right]\right\} \geq \frac{c_{10}}{n t} \exp \left[-2\left(1+\left(\frac{c_{9}}{2}\right) \varepsilon^{k+1}\right) \gamma_{L} \tau_{L} t^{1 / k}\right] .
$$

Thus, (17) and (18) imply that, for $\varepsilon \in(0,1), L \in G(d, k)$, and $\lambda_{1}$-almost all $t \geq 1$,

$$
\mathrm{P}\left\{\vartheta\left(Z_{0}(X \cap L), B_{L}\right) \geq \varepsilon \mid V_{k}\left(Z_{0}(X \cap L)\right)=t\right\} \leq c_{11} \exp \left[-c_{12} \varepsilon^{k+1} t^{1 / k}\right],
$$

where $c_{11}$ depends on $\gamma, \varphi$, and $\varepsilon$, and $c_{12}$ depends on $\gamma$ and $\varphi$. Thus, (16) yields the following theorem.

Theorem 5. Let $\varepsilon \in(0,1)$. There exist constants $c_{11}, c_{12}>0$, where $c_{11}$ depends only on $\varphi$, $\gamma$, and $\varepsilon$, and $c_{12}$ depends only on $\varphi$ and $\gamma$, such that

$$
\mathrm{P}\left\{\vartheta\left(Z_{0}^{(k)}, B_{L}\right) \geq \varepsilon \mid V_{k}\left(Z_{0}^{(k)}\right)=a, D\left(Z_{0}^{(k)}\right)=L\right\} \leq c_{11} \exp \left[-c_{12} \varepsilon^{k+1} a^{1 / k}\right]
$$

for $\mathrm{P}_{\left(V_{k}\left(Z_{0}^{(k)}\right), D\left(Z_{0}^{(k)}\right)\right)}$-almost all $(a, L) \in(1, \infty) \times G(d, k)$.

The exceptional set of $\mathrm{P}_{\left(V_{k}\left(Z_{0}^{(k)}\right), D\left(Z_{0}^{(k)}\right)\right)}$ measure zero that is possibly excluded in Theorem 5 can be chosen independently of $\varepsilon \in(0,1)$. This follows from the fact that the constant $c_{11}=c_{11}\left(\varphi, \gamma, \varepsilon^{\prime}\right)$ can be bounded uniformly for $\varepsilon^{\prime} \in[\varepsilon / 2, \varepsilon]$ for all $\varepsilon \in(0,1)$, as can be seen by an inspection of the proofs in [6].

\section{References}

[1] Baumstark, V. and Last, G. (2007). Some distributional results for Poisson-Voronoi tessellations. Adv. Appl. Prob. 39, 16-40.

[2] Hug, D. And Schneider, R. (2007). Asymptotic shapes of large cells in random tessellations. Geom. Funct. Anal. 17, 156-191.

[3] Hug, D. And Schneider, R. (2007). Typical cells in Poisson hyperplane tessellations. Discrete Comput. Geom. 38, 305-319.

[4] Hug, D. And Schneider, R. (2010). Large faces in Poisson hyperplane mosaics. Ann. Prob. 38, 1320-1344.

[5] Hug, D., Reitzner, M. And Schneider, R. (2004). The limit shape of the zero cell in a stationary Poisson hyperplane tessellation. Ann. Prob. 32, 1140-1167. 
[6] Hug, D., Reitzner, M. And Schneider, R. (2004). Large Poisson-Voronoi cells and Crofton cells. Adv. Appl. Prob. 36, 667-690.

[7] Kovalenko, I. N. (1997). Proof of David Kendall's conjecture concerning the shape of large random polygons. Cybernet. Systems Anal. 33, 461-467.

[8] Miles, R. E. (1964). Random polygons determined by random lines in a plane. II. Proc. Nat. Acad. Sci. USA 52, 1157-1160.

[9] Molchanov, I. (2005). Theory of Random Sets. Springer, London.

[10] Møller, J. (1989). Random tessellations in $\mathbb{R}^{d}$. Adv. Appl. Prob. 21, 37-73.

[11] SchneIder, R. (1993). Convex Bodies: the Brunn-Minkowski Theory. Cambridge University Press.

[12] Schneider, R. (2009). Weighted faces of Poisson hyperplane tessellations. Adv. Appl. Prob. 41, $682-694$.

[13] SchneIder, R. AND WeIL, W. (2008). Stochastic and Integral Geometry. Springer, Berlin.

[14] Stoyan, D., Kendall, W. S. And Mecke, J. (1995). Stochastic Geometry and Its Applications, 2nd edn. John Wiley, Chichester. 